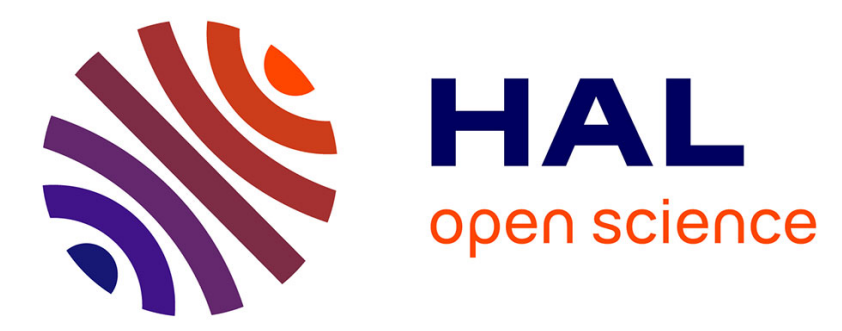

\title{
The evolution of an isolated turbulent region in a two-layer fluid
}

\author{
Jan-Bert Flór, Harindra J. Fernando, Gertjan F. van Heijst
}

\section{To cite this version:}

Jan-Bert Flór, Harindra J. Fernando, Gertjan F. van Heijst. The evolution of an isolated turbulent region in a two-layer fluid. Physics of Fluids, 1994, 6 (1), pp.287-296. 10.1063/1.868083 . hal01875873

\section{HAL Id: hal-01875873 \\ https://hal.science/hal-01875873}

Submitted on 24 Jan 2020

HAL is a multi-disciplinary open access archive for the deposit and dissemination of scientific research documents, whether they are published or not. The documents may come from teaching and research institutions in France or abroad, or from public or private research centers.
L'archive ouverte pluridisciplinaire HAL, est destinée au dépôt et à la diffusion de documents scientifiques de niveau recherche, publiés ou non, émanant des établissements d'enseignement et de recherche français ou étrangers, des laboratoires publics ou privés. 


\title{
The evolution of an isolated turbulent region in a two-layer fluid
}

\author{
J. B. Flor \\ J. M. Burgers Centre for Fluid Mechanics, Department of Technical Physics, \\ Eindhoven University of Technology, 5600MB Eindhoven, The Netherlands
}

\section{H. J. S. Fernando}

Program in Environmental Fluid Dynamics, Department of Mechanical and Aerospace Engineering, Arizona State University, Tempe, Arizona 85287-6106

G. J. F. van Heijst

J. M. Burgers Centre for Fluid Mechanics, Department of Technical Physics, Eindhoven University of Technology, 5600MB Eindhoven, The Netherlands

A turbulent region is geherated by horizontal pulsed injection at the interface of a two-layer fluid. Flow visualization studies reveal the existence of three stages in the evolution of the vertical size of this region: growth, maximum height, and collapse. A scaling analysis for the height of the turbulent region is presented, which appears to be in good agreement with the measurements. Comparable results were obtained by Fernando, van Heijst, and Fonseka (submitted to J. Fluid Mech.) for similar experiments in a linearly stratified fluid. Thorpe-scale measurements of the turbulent region reveal that the ratio of the rms displacement $L_{t}$ and the maximum displacement $L_{t \max }$ remain constant with time. The eventual formation process of the dipolar vortices after the collapse and the influence of interfacial wave motions on these dipolar vortices are discussed.

\section{INTRODUCTION}

In many geophysical flows stable stratification plays a major role in the evolution of turbulent flows. In such flows, vertical kinetic energy of fluid particles is converted into potential energy during their overturning motions, which leads to the suppression of vertical spreading of the turbulent region. After having reached its maximum vertical size, the mixed region starts to physically collapse under gravity until the motion is degenerated into a horizontal eddy pattern.

Local mixing events in stratified flows can occur by the breakdown of Kelvin-Helmholtz billows; some examples are the oceanic thermocline observations of Woods ${ }^{1}$ and Gregg, ${ }^{2}$ and the atmospheric observations of Browning. ${ }^{3}$ Other processes responsible for mixing events in stratified fluids are the breaking of internal waves, critical-layer absorption, and double diffusion. ${ }^{4}$

In the laboratory, the evolution of an isolated turbulent region in a linearly density-stratified fluid has been investigated by Fernando, van Heijst, and Fonseka, ${ }^{5}$ hereafter referred to as FvHF. In that study, an isolated turbulent region was generated by horizontally injecting a small volume of fluid during a short period of time, and the evolution of the emerging turbulent region was investigated. In this evolution three different stages have been identified: the turbulent region grows vertically (first stage) until it reaches its maximum size (the second stage), and subsequently collapses under gravity (third stage). The flow eventually is transformed into a dipolar vortex structure, as was described by van Heijst and Flór ${ }^{6}$ and Voropayev et al. ${ }^{7}$ For each stage a scaling analysis was given for the evolution of the vertical patch size.

The purpose of the present investigation was to extend the work of FvHF to a two-layer fluid; i.e., the pulsed injection of fluid takes place at the interface of a two-layer fluid. Although the details are different, the qualitative features of the evolution in linearly stratified and two-layer fluids appear to be the same. In the present study, flow visualization and measurements of the vertical density distributions are tested to study the evolution of the injected turbulent region. The experimental arrangement for these measurements is described in Sec. II. Qualitative observations are described in Sec. III, and the experimental results as well as scaling analyses of the growth and the subsequent collapse of the turbulent region are presented in Sec. IV. Additional measurements on the dipolar vortex structure that is formed at the later stages are discussed in Sec. V. A brief summary of the results and conclusions are given in Sec. VI.

\section{EXPERIMENTAL SETUP}

The experiments reported in this paper were conducted in two different Perspex tanks: one narrow tank of dimensions $183 \times 30 \times 40(\mathrm{~cm})$ and one square tank of dimensions $90 \times 90 \times 40(\mathrm{~cm})$, respectively. For the study of the growth and collapse of the turbulent patch, the narrow tank was used, while the square tank was used to study the dipole formation. In order to assure that side wall effects in the small tank did not bias the collapse significantly, the collapse stage was studied in the large tank. A schematic of the experimental arrangement is shown in Fig. 1. The test cell was filled with two layers of fluid, the upper layer containing fresh water and the lower layer containing a salt solution. The stratification was established by carefully adding the salty water underneath the fresh layer. In order to obtain a sharp interface, fluid from the center of the 


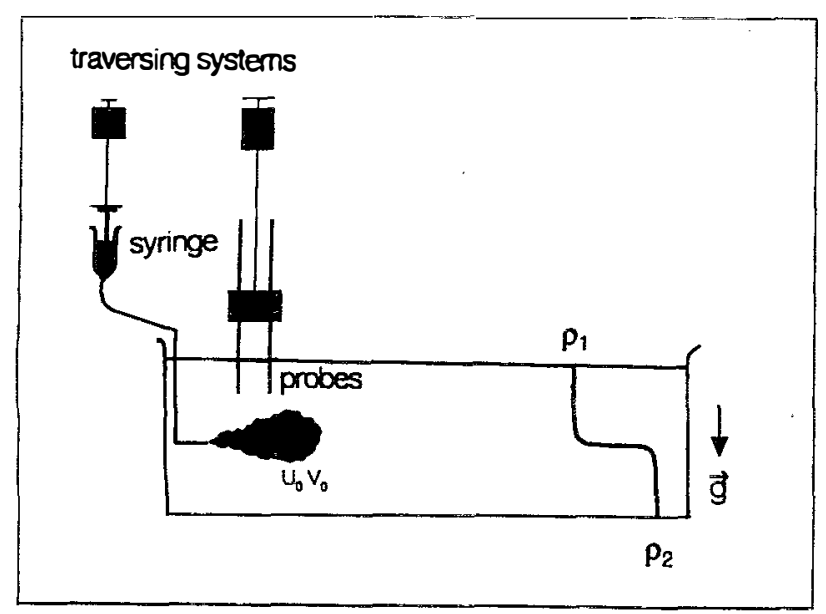

FIG. 1. A sketch of the experimental setup.

interface was siphoned off using small perforated horizontal tubes. By this method the interface thickness $\delta h$ was reduced to a thickness of approximately $0.6-2.5 \mathrm{~cm}$, which was always smaller than the size of the turbulent patch. The total depth of the water was varied from 20 to $31 \mathrm{~cm}$, and the position of the interface, measured from the bottom of the tank, was varied between 12 and $14 \mathrm{~cm}$. The buoyancy jump across the interface, represented by

$$
\delta b=2 g \frac{\rho_{2}-\rho_{1}}{\rho_{1}+\rho_{2}},
$$

with $\rho_{1}$ and $\rho_{2}$ the densities of the upper and lower layer, respectively, and $g$ the gravitational acceleration, was in the range from 0.37 to $0.77 \mathrm{~m} / \mathrm{s}^{2}$. In order to measure the density profile a conductivity probe was traversed vertically through the fluid, from which the interfacial position was determined. Then the nozzle was placed at this level and a small drop of the dyed injection fluid-with density $\left(\rho_{1}+\rho_{2}\right) / 2$-was released at the level of the nozzle. Due to the strong buoyancy force at the interface the drop collapses instantly to a thin spot of less than a millimeter thickness and marked the midlevel of the interface quite accurately. The fluid was injected horizontally at this level through a nozzle of diameter $2 \mathrm{~mm}$ by a computercontrolled injection mechanism. The density of the injected fluid was adjusted to the intermediate density, $\left(\rho_{1}+\rho_{2}\right) / 2$. The injection velocity in these experiments was varied from 1.8 to $11.7 \mathrm{~m} / \mathrm{s}$, and the injection volume $V_{0}$ ranged from 1 to $13 \mathrm{ml}$. The time of injection $\delta t$ was varied between $0.01-0.2 \mathrm{~s}$, which was sufficiently short to create an isolated turbulent patch of fluid. The Reynolds number $\operatorname{Re}=U_{0} d / v$ at the injection, where $U_{0}$ is the injection velocity, $d$ is the nozzle diameter, and $v$ is the kinematic viscosity, ranged from 3500 to 22000 , thus ensuring that the jet was turbulent. The resulting turbulent patch was visualized by coloring the injected fluid with fluorescein and by illuminating it using a vertical sheet of light. The extent of dye diffusion was considered as the extent to which the turbulence diffuses; that is, the turbulent Prandtl number is considered to be of the order unity (Refs. 8 and
9). A horizontal light sheet was used for visualizing the dipolar vortex formation in the square tank. The evolution of the flow was recorded by a video camera and by a frameby-frame examination of these recordings the time evolution of the vertical patch size was determined. The moment at which the first spot of colored fluid is ejected by the nozzle, was taken as time $t=0$.

The vertical density distribution was measured by using a fast traversing microscale conductivity probe that was installed above the square tank. This traversing mechanism could be translated horizontally to track and capture the moving patch during the course of an experiment. The plunging speed of the probe had a maximum value of 30 $\mathrm{cm} / \mathrm{s}$, which is fast compared with the observed maximum translation speed of the patch (about $5 \mathrm{~cm} / \mathrm{s}$ ). These instantaneous vertical density profiles were used to calculate Thorpe displacements, i.e., the size of the vertical displacements of fluid parcels relative to a stable profile, which was obtained by rearranging the observed density profile. The method employed is similar to that used by Dillon. ${ }^{10}$ The time and position at which the probes were shot, were recorded by video.

\section{QUALITATIVE OBSERVATIONS}

The evolution of the turbulent patch after the injection is presented in Fig. 2. During and shortly after the injection, an approximately cone-shaped jet forms and evolves into an isolated three-dimensional turbulent patch and moves in the direction of injection. During the initial stage, the vertical inertia forces of the eddies are large compared to the buoyancy forces, and entrainment of homogeneous fluid from above and below the interface causes the turbulent region to grow continuously [see Fig. 2(b)]. Mixing of jet fluid and ambient fluid implies that the kinetic energy is converted into potential energy. When the vertical inertia forces become of the same order as the buoyancy forces, the turbulent patch reaches its maximum vertical size and then starts to collapse under gravity, thus reducing its vertical size [for example, see Fig. 2(c)]. During the collapse, the mixed fluid intrudes horizontally into the ambient fluid. In some cases one could observe the formation of two separated intrusion noses at the front of the intrusion: one above and one below the interface [see, e.g., Figs. 2(c) and $2(d)]$. The occurrence of the double intrusion can be attributed to incomplete mixing within the patch, due to the finite thickness of the initial interface, ${ }^{11}$ as well as interfacial friction between the two intrusions. The collapse continues until the patch attains a thickness comparable to that of the interface. Interfacial waves, generated during the collapse, could not be distinguished from the waves that were forced by the initial impulse of the jet. In most of the experiments it is observed that these waves move away from the turbulent region along the upper and lower boundaries of the intrusion. These traveling waves are dissipated rapidly, in particular, after having been reflected at the tank walls. From the video recordings, however, it appears that in some cases a soliton-like disturbance moves at the front of the patch, giving rise to a vertical bulge near the nose of the intrusion. This bulge vanishes slowly com- 


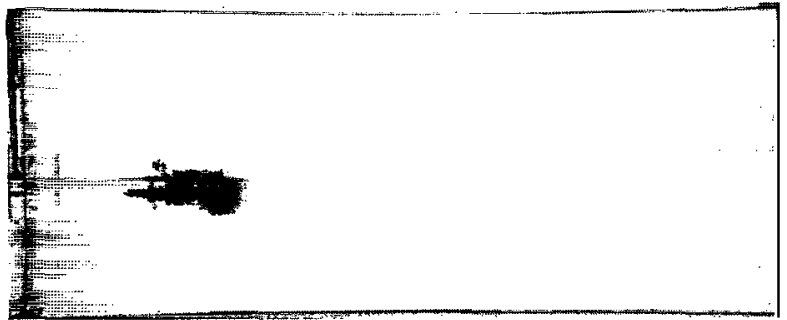

(a)

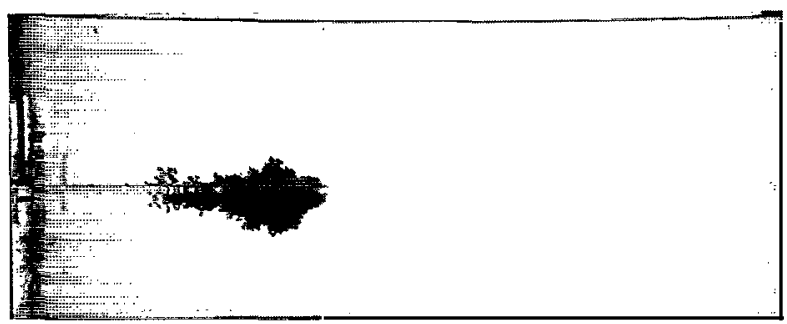

(b)

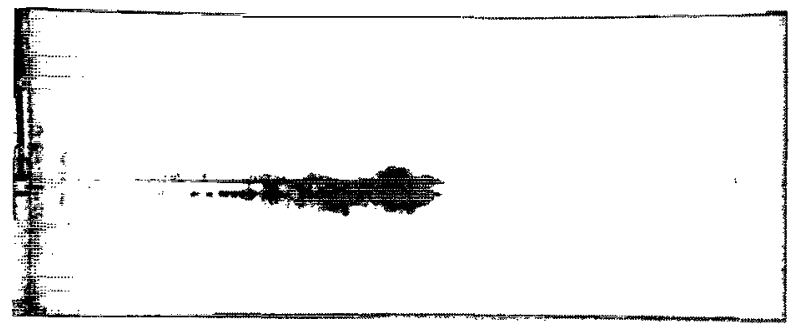

(c)

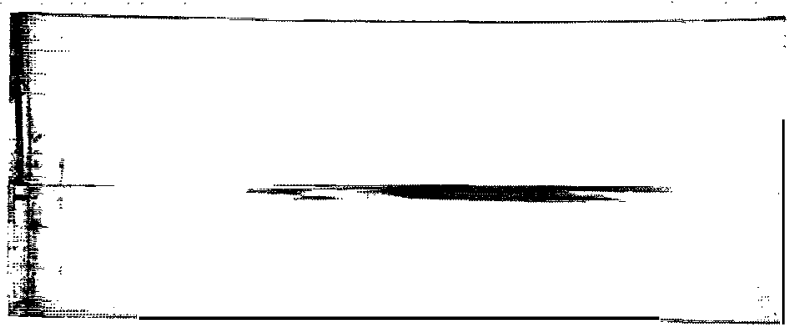

(d)

FIG. 2. A sequence of photographs, showing the evolution of the turbulent region as viewed from the side. A volume of fluid $V_{0}=2 \mathrm{ml}$ is injected horizontally at the level of the interface with $U_{0}=3.16 \mathrm{~m} / \mathrm{s}$ and $\delta b=0.60$ $\mathrm{m} / \mathrm{s}^{2}$. The pictures are taken at (a) 0.44 , (b) 1.0 , (c) 1.44 , and (d) 44.8 $s$ after the injection. The top-bottom distance in each picture represents the scale and is $30 \mathrm{~cm}$.

pared to the collapse rate of the patch. In a few experiments with high injection velocities, a soliton-like feature was observed to detach from the collapsing region and travel up and down the tank a few times (see Fig. 3). Similar wave motions have been observed by Gilreath and Brandt ${ }^{12}$ in the wake of an obstacle towed through a density interface. At the earlier stages of the collapse, the intrusion front is contorted and is observed to be distorted by flattened large eddies, which persist for longer times than the small-scale turbulence. At later stages, the front changes into one single, sharp intrusion. After the collapse the motion is mainly horizontal. A dipolar vortex structure emerges, which moves slowly in a straight line along its axis of symmetry. This dipolar structure takes on the same

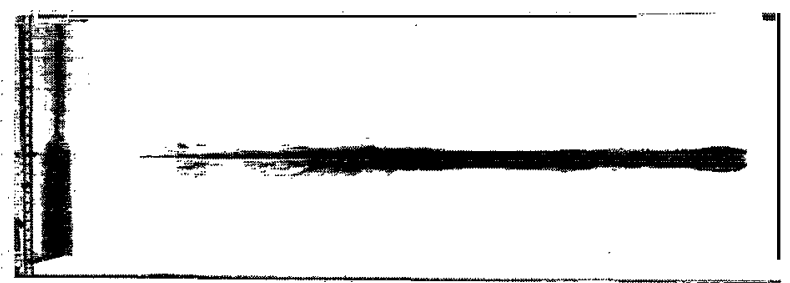

(a)

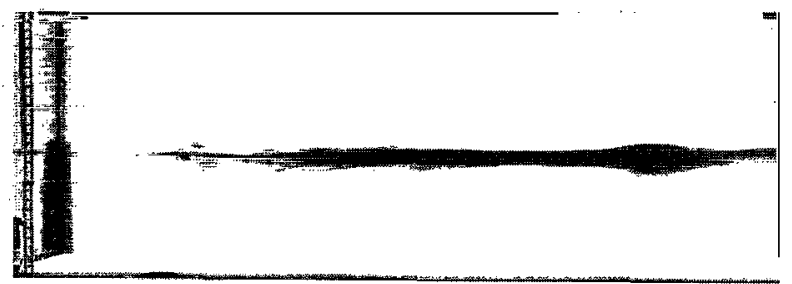

(b)

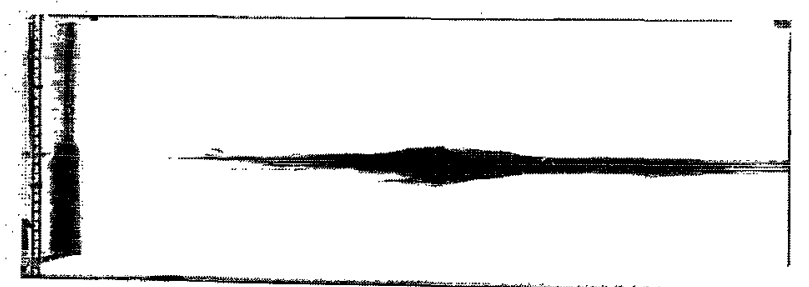

(c)

FIG. 3. Soliton-like disturbance at the nose of the intrusion just before separation from the intrusion (a) at $t=9.6 \mathrm{~s}$, after separation and collision with the wall (b) at $t=17.0 \mathrm{~s}$, and at $t=23.4 \mathrm{~s}$. The experimental parameters for this experiment were $\delta b=0.615 \mathrm{~m} / \mathrm{s}^{2}, U_{0}=3.6 \mathrm{~m} / \mathrm{s}$, and $V_{0}=16.8 \mathrm{ml}$. (This particular experiment was carried out later in Eindhoven with a less powerful injection mechanism, which allowed only for smaller injection velocities. To obtain the same collapse height and therewith a similar soliton, a larger volume had to be injected.) The dimensions of the tank used for this visualization study were $90 \times 40 \times 40 \mathrm{~cm}$. The top-bottom distance in each picture represents the scale, which is $27 \mathrm{~cm}$.

appearance as the dipoles observed in a linearly stratifed fluid by van Heijst and Flór, ${ }^{6}$ Voropayev et al., ${ }^{7}$ and FvHF.

Typical examples of the vertical density profile through a turbulent patch in a linearly stratified fluid and in a two-layer fluid are presented in Figs. 4(a) and 4(b), respectively. In a linearly stratified fluid, the turbulent eddies over the entire patch are competing with roughly the same restoring buoyancy force, which results in a single mixed region [see Fig. 4(a)]. For the two-layer case, the entrained fluid from the upper and lower layers have a buoyancy $\delta b / 2$ with respect to the turbulent eddies in the patch. The entrained lighter fluid from the upper layer will be carried downward and the heavy fluid (entrained from the lower layer) will be carried upward by the overturning fluid of density $\left(\rho_{1}+\rho_{2}\right) / 2$. Initially, the lighter fluid tends to concentrate in the upper part of the patch, and the heavier fluid will be in the low part of the patch [see Fig. 4(b)]. When eddies continue to overturn fluid across the midplane, they will experience an almost twofold increase of the buoyancy force, which may suppress the overturning if the inertia forces of the eddies are too weak to perform overturning motions against the increased buoyancy 

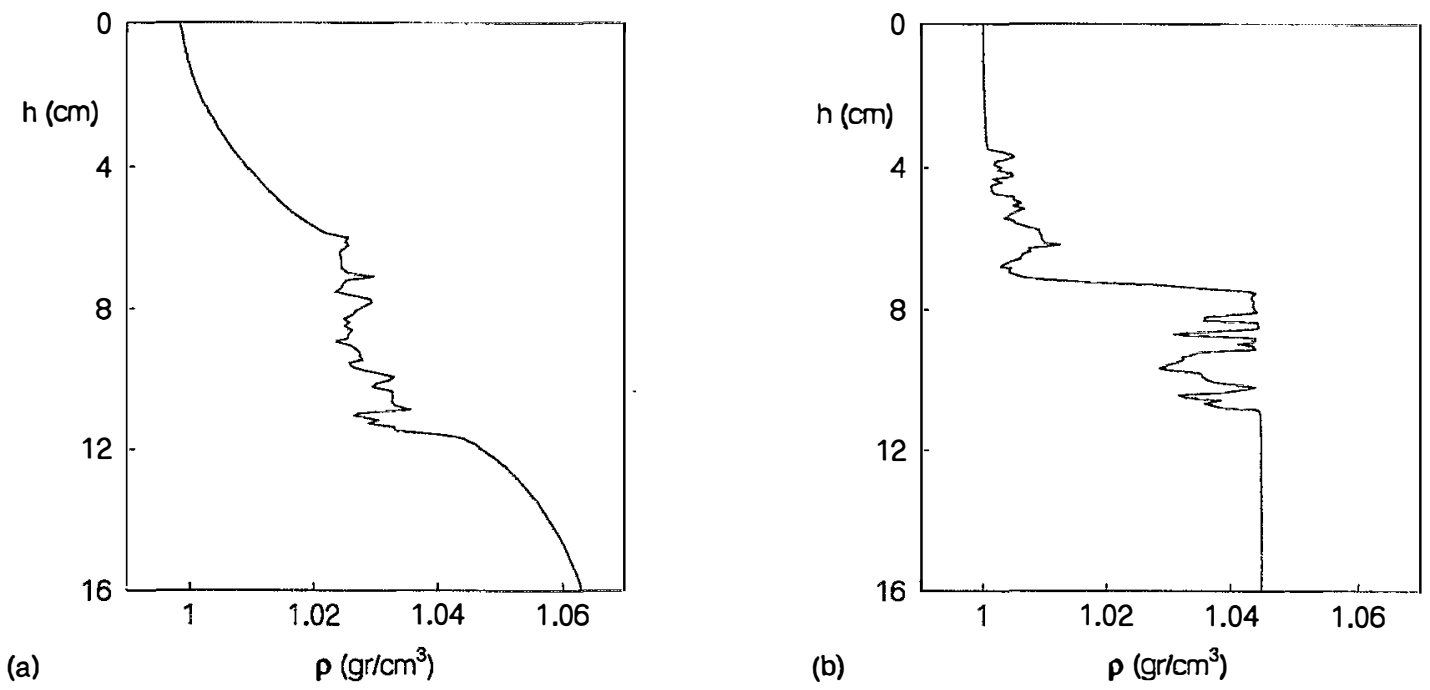

FIG. 4. Density profile of the turbulent patch before the onset of collapse in (a) a continuous ambient stratification and in (b) a two-layer fluid. The stratification parameters are the Brunt-Väisälä frequency $N=[(g / \rho)(\partial \rho / \partial z)]^{1 / 2}=2.2 \mathrm{rad} / \mathrm{s}$ for (a) and $\delta b=0.48 \mathrm{~m} / \mathrm{s}^{2}$ for (b). The profiles were measured at (a) $t=0.9 \mathrm{~s}$ and (b) $1.4 \mathrm{~s}$ after injection.

forces. During the collapse, both mixed regions give rise to two different intrusions, as observed from flow visualizations [see Figs. 2(c) and 2(d)]. When the dipolar vortex structure emerges, only the horizontal motions remain at the interface and due to molecular diffusion between the sharp intrusions, an intrusion with a single nose appears. In some experiments it was observed that remnant standing interfacial wave motions interact with the dipole vortices. In contrast to the traveling internal waves, these waves decay with a much longer time scale. The standing internal waves induce pulsations in vortex size, which cause stretching and compression of the vortices. This mechanism leads to changes in the vertical vorticity component of the dipolar structure. As a consequence, the translation velocity and the horizontal size of the dipolar structure show oscillations around their mean values. Eventually, these standing waves are damped out and the dipole translates at a low velocity.

\section{COLLAPSE OF THE TURBULENT REGION}

For a typical experiment, the maximum vertical patch size and the maximum horizontal spreading of the region were measured from video recordings. The vertical patch size as a function of time is shown in Fig. 5 (a), whereas the combination of the maximum horizontal and vertical patch sizes is presented in Fig. 5(b). Initially, the turbulent region grows equally in horizontal and vertical directions due to entrainment of ambient fluid. Once the influence of the buoyancy forces sets in, the patch growth is retarded
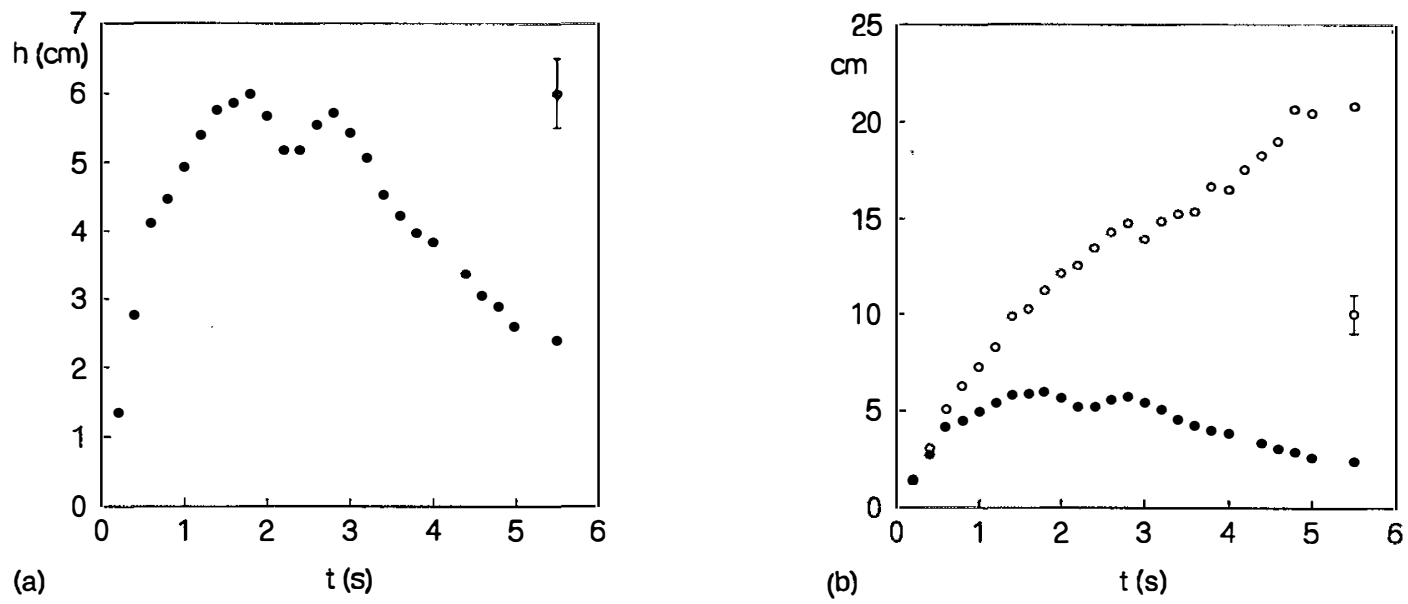

FIG. 5. Typical evolution of (a) the vertical patch size and (b) the corresponding growth in horizontal size compared to the vertical growth. The vertical patch size is denoted by the symbol $\bigcirc$ and the horizontal patch size by the symbol $\bullet$. The experimental parameters for this experiment were $\delta b=0.757$ $\mathrm{m} / \mathrm{s}^{2}, U_{0}=5.44 \mathrm{~m} / \mathrm{s}$, and $V_{0}=11.1 \mathrm{ml}$. The dimensions of the tank used for this visualization study were $300 \times 40 \times 40 \mathrm{~cm}$. 
and finally reaches its maximum size. Subsequently, the patch collapses and decreases in vertical size while the horizontal dimensions increase continuously with approximately the same growth rate as before the collapse [see Fig. 5(b)]. For all the experiments the vertical size of the patch is measured, until changes were no longer noticeable, i.e., when the horizontal size is approximately $15 \mathrm{~cm}$, and the influence of the tank width is still negligible. The collapse continues until the patch is confined in a thin layer of fluid, and pancake-like eddies start to form. In some cases it was observed that interfacial wave motions cause the mixed patch to oscillate with a period of about $0.5-1 \mathrm{~s}$. These wave motions induce oscillations in the vertical patch size during the growth and subsequent collapse [see Fig. 5(a)]; these, however, are considered to be of minor importance to the evolution of the vertical patch size, and are thus neglected in the scaling analysis, which will be discussed below.

\section{A. The growth of the turbulent region}

During the initial growth, the turbulent motions are dominated by the inertia forces. Thus, a scaling analysis similar to that advanced by FvHF for the growth of a turbulent region in a linearly stratified fluid can be used. The growth of the turbulent region lasts for a very short period of time, so that the kinetic energy during that period can be assumed to be a constant, viz.,

$$
\overline{u^{\prime 2}} \sim \frac{V_{0} U_{0}^{2}}{V(t)},
$$

where $U_{0}$ is the injection velocity, $V_{0}$ is the injected volume at time $t=0$, and $\overline{u^{\prime 2}}(t)$ and $V(t)$ are the mean square horizontal turbulent velocity and volume of the turbulent region at time $t$, respectively. During the evolution, the turbulent region can be approximated by a self-similar conical shape of characteristic dimension $h(t)$, and thus its volume can be approximated by $V(t) \sim h(t)^{3}$. For the turbulence within the turbulent region it is assumed that $\overline{u^{\prime 2}}$ $\sim{\overline{w^{\prime 2}}}$, with ${\overline{w^{\prime 2}}}^{1 / 2}$ being the rms vertical velocity. Using the scaling for $\overline{u^{\prime 2}}$ and $V(t)$, one may write

$$
\overline{w^{\prime 2}}=B_{1} \frac{V_{0} U_{0}^{2}}{h^{3}},
$$

where $B_{1}$ is a constant. Since the initial growth is dominated by inertial forces of turbulence, it is possible to model the entrainment velocity as ${ }^{8}$

$$
\frac{d}{d t} h=B_{2}{\overline{w^{\prime 2}}}^{1 / 2},
$$

similar to what has been done in previous related studies (Refs. 13 and 14). Although (4) is valid strictly for forced turbulent flows, based on the findings of Mobbs, ${ }^{15}$ it is assumed that it is also applicable for decaying turbulent patches. Then it is possible to obtain, using (3) and (4), a relation for the growth as a function of time,

$$
\frac{h(t)}{V_{0}^{1 / 3}}=B_{3}\left(\frac{U_{0}^{6} t^{6}}{V_{0}^{2}}\right)^{1 / 15} \text {, }
$$

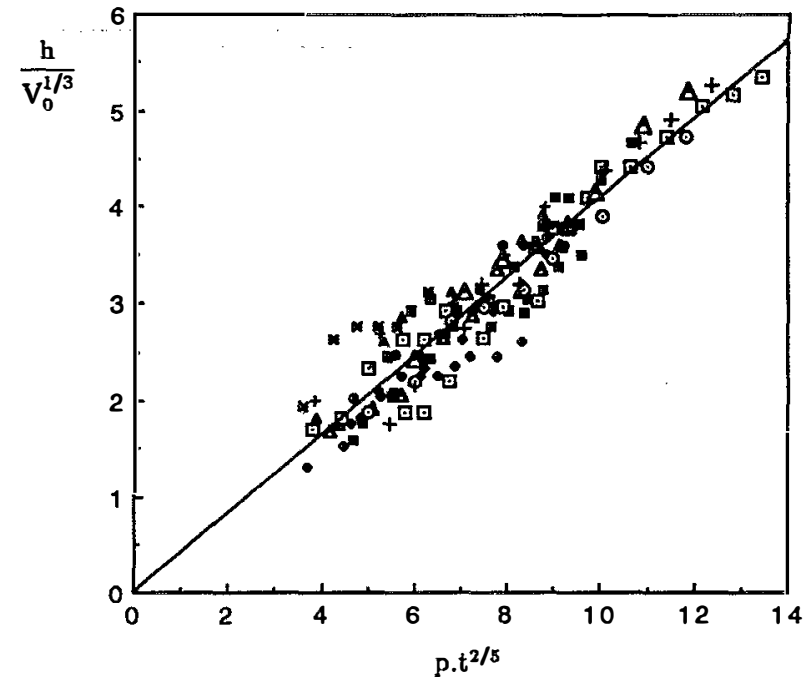

FIG. 6. The normalized vertical size of the turbulent region as measured for 16 experimental runs, with $p=\left(U_{0}^{0} / V_{0}^{2}\right)^{1 / 15}$. The experimental parameters and the proportionality constant $B_{3}$ for each individual experiment are given in Table I.

where we have assumed $h(0) \ll h(t)$, and $B_{3}=\left(\frac{5}{2} B_{2} B_{1}^{1 / 2}\right)^{2 / 5}$. Normalized data for the initial growth for 15 experiments are presented in Fig. 6, which show good agreement with (5). The least-square fit of the data yields $B_{3}=0.40 \pm 0.06$. This value is comparable with the corresponding constant $A_{3}=0.37$ for the growth of a turbulent jet in a linearly stratified fluid, as measured by FvHF. The agreement between the two values reveals that the stratification does not substantially influence the initial growth of the turbulent region.

\section{B. The maximum patch size}

The growth of the turbulent blob continues until the vertical inertia forces $\left(\overline{w^{\prime 2}} / h\right.$ per unit mass $)$ of the eddies have decreased to such an extent that the buoyancy forces ( $\delta b$ per unit mass) become of comparable magnitude. At that stage, the patch reaches its maximum vertical size, and the inertia forces are balanced by the buoyancy forces, viz.,

$$
h_{c} \delta b=B_{4} \overline{w^{\prime 2}},
$$

where $h_{c}$ is the maximum vertical size of the patch. In other words, this relationship implies that the patch collapse occurs when the bulk Richardson number $h_{c} \delta b / \overline{w^{\prime 2}}$ increases to a critical value $B_{4}$. By using (3) and (6), it is possible to obtain

$$
h_{c}=B_{5}\left(\frac{V_{0} U_{0}^{2}}{\delta b}\right)^{1 / 4} \text {, }
$$

where $B_{5}=\left(B_{1} B_{4}\right)^{1 / 4}$. The experimental results for the maximum patch height are presented in Fig. 7, and it is obvious that they show good agreement with the proposed scaling. The best fit required for the data yields $B_{5}=0.5$. By using (5) and (7) the time $t_{c}$, at which the patch reaches its maximum size $h_{c}$, can be written as 


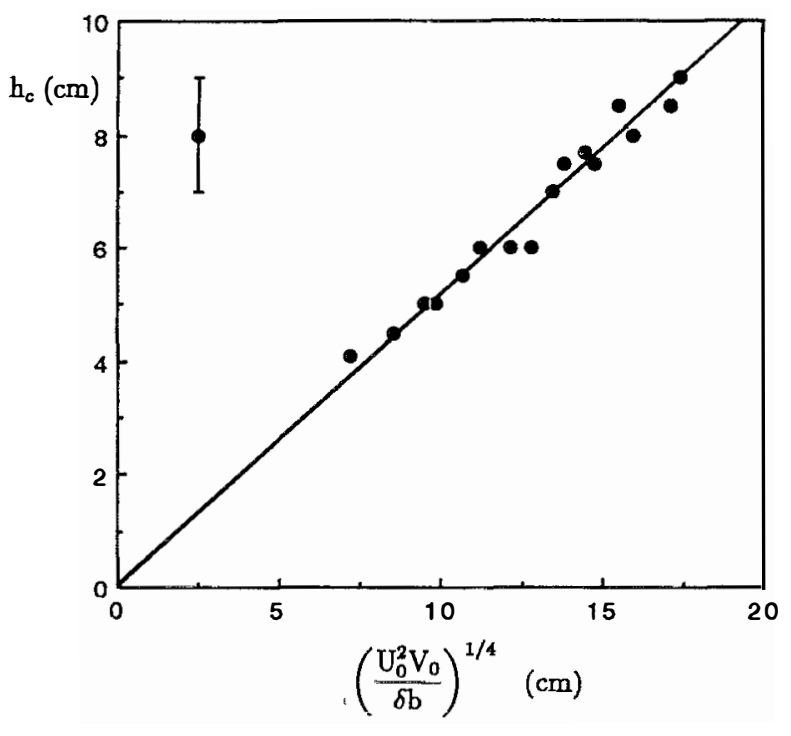

FIG. 7. The maximum vertical size of the turbulent region as measured in 16 experimental runs. The experimental parameters are presented in Table I.

$$
i_{c}=B_{6}\left(\frac{V_{0} U_{0}^{2}}{\delta b^{5}}\right)^{1 / 8},
$$

where $B_{6}=\left(B_{5} / B_{3}\right)^{5 / 2}=1.7$ is obtained by using $B_{3}$ and $B_{5}$. Direct measurements of the collapse time $t_{c}$ from the experiments yields a value of $B_{6}=2.2 \pm 0.6$. This disparity can be attributed to the difficulty of locating the time $t_{c}$ to attain the maximum $h_{c}$. Nevertheless, the constants are of the same order, showing a reasonable agreement of the scaling arguments with the measurements.

\section{The collapsing stage}

After the patch has reached its maximum height, the eddies of the integral scale are too feeble to entrain the ambient fluid. An isolated patch of mixed fluid with a density different from the surrounding fluid remains, while the turbulence within the patch is weakened and finally is transformed into small-scale wave-like motions. Simple hydrostatic calculations show that there will be a horizontal pressure gradient between the inner and outer fluid, which causes the mixed patch to physically collapse and the mixed fluid to intrude in the ambient fluid at rest. The speed of the intrusion can be evaluated by a balance between the pressure gradient force and the inertia force of the intrusion: ${ }^{16}$

$$
\frac{d r}{d t}=C_{1}(h \delta b)^{1 / 2},
$$

where $h(t)$ and $r(t)$ are the total vertical patch size and the horizontal dimension, respectively, and $C_{1}$ is a constant. Assuming a double conical shape for the mixed patch, the horizontal spreading rate $d r / d t$ can be estimated by

$$
h_{c}^{3}=2 r^{2} h(t) \text {. }
$$

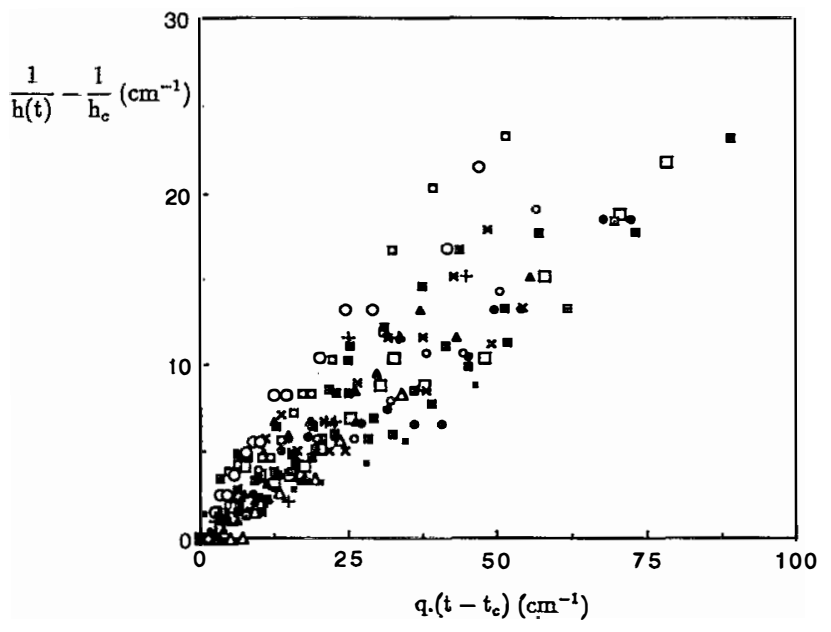

FIG. 8. The experimental results for the collapse stage plotted in the form of Eq. (11), with $q=\left(\delta b^{7} / V_{0}^{3} U_{0}^{6}\right)^{1 / 8}$. The experimental parameters are given in Table I.

By using (9), (10), and the expression for the maximum vertical patch size (7), it is possible to obtain

$$
\frac{1}{h}-\frac{1}{h_{c}}=C_{3}\left(\frac{\delta b^{7}}{V_{0}^{3} U_{0}^{6}}\right)^{1 / 8}\left(t-t_{c}\right),
$$

where $C_{3}=2 \sqrt{2} B_{5}^{-3 / 2} C_{1}$. A comparison of data with (11) is presented in Fig. 8. The values of $C_{3}$ for individual experiments are listed in Table I. The constant $C_{3}=0.30 \pm 0.09$ is obtained by taking the mean value of $C_{3}$ over all measurements. Although the data for each individual experiment are in good agreement with the proposed scaling, there is some spread of the values of $C_{3}$ over all the experiments. This is probably due to the interaction of the turbulent patch with interfacial wave motions, and the neglect of the development of the density gradient within the patch during initial mixing. The value $C_{1}$ can be evaluated by using $C_{3}$ and yields $C_{1}=0.04$.

\section{The Thorpe-scale measurements}

In a turbulent stratified fluid heavy fluid elements are displaced above the lighter ones by turbulent stirring motions, causing density inversions. One method to estimate the scale of such overturning motions is to determine the vertical displacements of fluid elements relative to a stable, monotonic density profile. This density profile can be obtained by rearranging the measured instantaneous profile into a stable one by displacing fluid particles to their neutral buoyancy depth by a so-called "bubble sort" routine. ${ }^{10}$ The required displacements are called Thorpe displacements, which provide information about the overturning motions of the eddies. The rms value of these displacements is known as the Thorpe length scale $L_{t}$.

In order to measure the Thorpe scale of a decaying turbulent region, a number of experiments were performed under identical conditions, i.e., the same injection velocity and volume, and stratification. For each experiment, two traversing conductivity probes-with a distance $2.9 \mathrm{~cm}$ 
TABLE I. Table of the injection parameters and the evaluated constants of the laboratory experiments. $U_{0}, V_{0}$, and $\delta b$ are the injection velocity, injection volume, and buoyancy jump across the interface, respectively. The experimental constants for the initial growth and the collapse $B_{3}$ and $C_{3}$, respectively (see the text) are given for each experiment, together with their correlation coefficients $R^{2}$ of the approximations with the measurements. For experiments 20-22 a soliton-like wave motion occurred, and no regular collapse was observed.

\begin{tabular}{|c|c|c|c|c|c|c|c|}
\hline $\exp$ & $U_{0}(\mathrm{~m} / \mathrm{s})$ & $V_{0}(\mathrm{ml})$ & $\delta b\left(\mathrm{~m} / \mathrm{s}^{2}\right)$ & $B_{3}$ & $R^{2} B_{3}$ & $C_{3}$ & $R_{--}^{2} C_{3}$ \\
\hline 8 & 5.82 & 5 & 0.779 & 0.400 & 0.922 & 0.401 & 0.976 \\
\hline 15 & 2.92 & 7 & 0.653 & 0.268 & 0.885 & 0.251 & 0.976 \\
\hline 16 & 11.71 & 4 & 0.653 & 0.493 & 0.965 & 0.430 & 0.971 \\
\hline 18 & 9.08 & 5 & 0.653 & 0.433 & 0.935 & 0.360 & 0.904 \\
\hline 21 & 5.78 & 1 & 0.653 & 0.374 & 0.975 & & \\
\hline 22 & 5.78 & 11 & 0.653 & 0.370 & 0.947 & & \\
\hline 23 & 3.21 & 5 & 0.653 & 0.439 & 0.952 & 0.2 & 0.884 \\
\hline 24 & 10.81 & 5 & 0.653 & 0.501 & 0.984 & 0.155 & 0.934 \\
\hline 25 & 1.8 & 3 & 0.371 & 0.36 & 0.797 & 0.250 & 0.965 \\
\hline 26 & 6.4 & 3 & 0.371 & 0.456 & 0.981 & 0.286 & 0.949 \\
\hline
\end{tabular}

apart-were positioned at a given distance from the injection nozzle. These probes were shot vertically downward through the fluid at the instant that the turbulent region was at the same position as the probes. Information about the spatiotemporal evolution of the turbulent region was obtained by changing the position of the probes for each experiment. By using the measured density profiles, the maximum Thorpe displacement $L_{t \max }$, the Thorpe scale $L_{t}$, and the vertical patch size $L_{p}$ were determined: $L_{p}$ was defined as the region that has displacements exceeding $5 \%$ of $L_{t \max }$. This threshold of $5 \%$ was necessary to suppress the noise peaks generated at the start and end position of the probe traverse. In addition, the patch size $h$ was measured using video records, and the results are shown in Fig. 9. The latter showed consistently higher values. This is not unexpected, in view of the definition of the patch size $L_{p}$ as being the region that contains Thorpe displacements larger than $5 \%$ of $L_{t \max }$. Outside this region, overturning smallscale motions can prevail and can disperse dyed fluid. Dur-

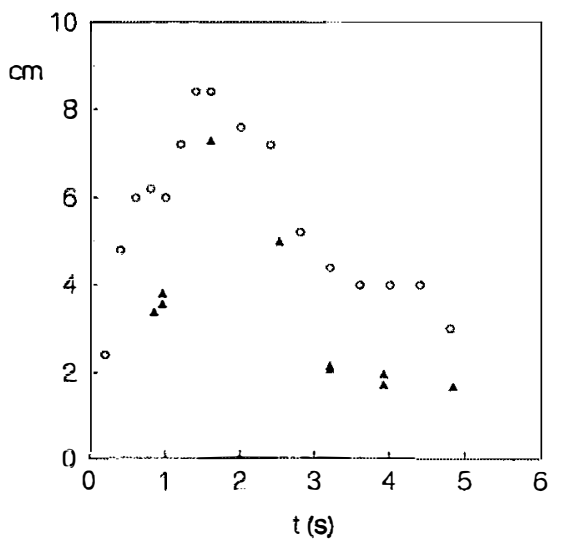

FIG. 9. A comparison of the decay of the vertical patch size $L_{p}$ obtained from Thorpe displacements and the patch size $h$ from video recordings, denoted by the symbols $\boldsymbol{\Delta}$ and $O$, respectively. ing the collapse of the patch the difference between these two patch size measurements becomes larger. Under these conditions, the color visualization technique indicates the physical size of the collapsing region, whereas the Thorpe displacement technique indicates the return of buoyant fluid parcels to their respective stable position. The latter occurs faster, as was indicated by the measurements of FvHF.

The Thorpe scale $L_{t}$ and the nondimensional Thorpe scale $L_{t} / L_{t \max }$ are plotted against time in Fig. 10. The graph shows that $L_{t}$ decays approximately linearly with time. The normalized Thorpe scale $L_{t} / L_{t \max }$ appears to remain constant ( $\simeq 0.4$ ) during the evolution of the patch. This is in fair agreement with the previous observations of Itsweire ${ }^{17}$ and DeSilva and Fernando, ${ }^{18}$ which showed $L_{t \max } / L_{t} \simeq 2.75$, for decaying stratified turbulence and forced stratified turbulence, respectively. Shown in Fig. 11 is a plot of $L_{t}$ vs $L_{p}$; a linear increase can be seen with $L_{t}$

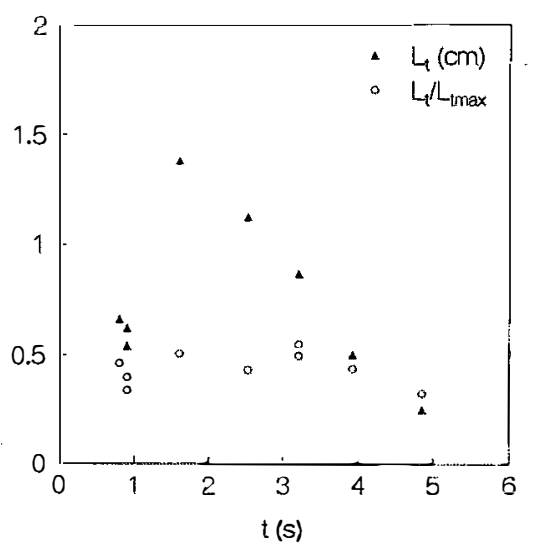

FIG. 10. The Thorpe scale $L_{t}$ and the nondimensionalized Thorpe scale $L_{t} / L_{t \max }$ against time, denoted by the symbols $\Delta$ and $\bigcirc$, respectively. The data were obtained in an experiment with $U_{0}=5.96 \mathrm{~m} / \mathrm{s}, V_{0}=4.5 \mathrm{ml}$, and $\delta b=0.53 \mathrm{~m} / \mathrm{s}^{2}$. 


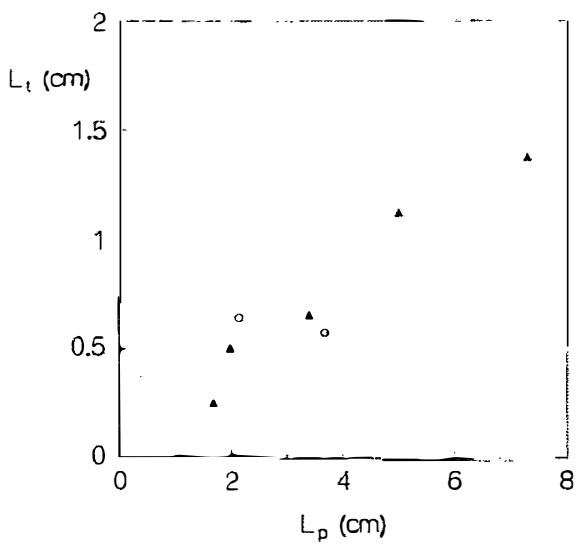

FIG. 11. The variation of the Thorpe scale $L_{t}$ plotted against the patch size $L_{p}$. The symbol $O$ refers to averaged values of two probes.

approximately equal to $0.18 L_{p}$. Inspection of Fig. 4 shows that the maximum overturning scale (or the maximum Thorpe displacement $L_{t \max }$ ) cannot exceed $L_{p} / 2$ as a result of the development of the jump in the stratification at the mid-depth of the patch. The above measurements also show $L_{t \max }=0.18 / 0.4 \simeq 0.45 L_{p}$. Gibson ${ }^{19}$ postulated that the patch size should be of the same order as the maximum Thorpe displacement. This notion is consistent with our experimental results, because in our case the effective patch size is half the observed value, in view of the development of a sharp density gradient at the middle of the patch. Thus, the effective ratio is $L_{t \max } / L_{p}=0.45 /\left(L_{p} / 2\right) \simeq 0.9$. This value is somewhat higher than that of DeSilva and Fernando, ${ }^{18}$ who found $L_{t \max } / L_{p} \simeq 0.75$ for continuously stirred patches.

\section{ADDITIONAL OBSERVATIONS; THE DIPOLAR STRUCTURE}

During the collapse stage, mixed fluid is spread radially outward at its equilibrium density level until a flat region of fluid with only horizontal motions remains [see Fig. 12(a)]. By continuity of mass this diverging flow of mixed fluid induces a low pressure in the center of the collapsed region. After the collapse, it is observed that the intruded fluid flows back to this center of collapse. At this stage larger horizontal vortices are seen to arise, and their merger leads to the formation of the dipolar vortex structure. Simultaneously, the thickness of the dipole becomes larger than that of the flat collapsed region [see Figs. 12(a) and 12(b)]. This dipolar vortex structure has an appearance similar to the dipoles observed in linearly stratified fluids (see Refs. 6 and 7).

The dipolar vortex in a two-layer case is significantly affected by internal waves traveling along the interface. These internal waves are mainly generated by the momentum of the initial injection and cause oscillations in size and vorticity amplitude of the dipolar vortex. Waveinduced stretching and squeezing of vertical vortex tubes within the dipolar structure cause a continuous changing of the vertical vorticity components of the vortex structure,

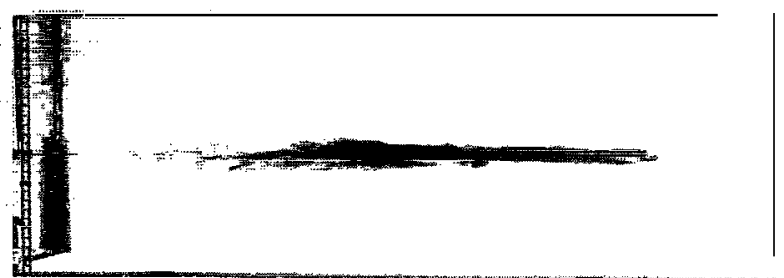

(a)

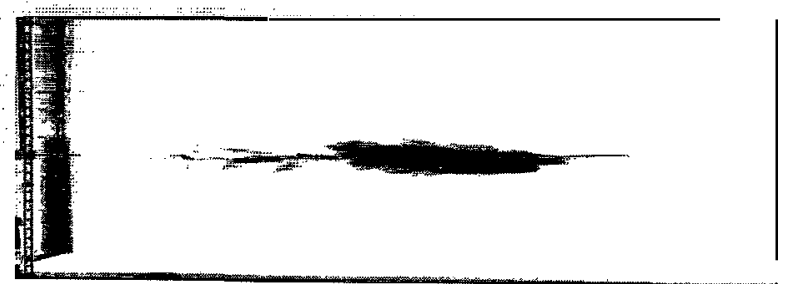

(b)

FIG. 12. Sideview photographs of the collapsed region (a) before and (b) after the emergence of the larger vortex structure. The photographs were taken at (a) $t=9.0 \mathrm{~s}$ and (b) $t=20.0 \mathrm{~s}$, respectively. The experimental parameter values are $\delta b=0.615 \mathrm{~m} / \mathrm{s}^{2}, U_{0}=3.6 \mathrm{~m} / \mathrm{s}$, and $V_{0}=13.4 \mathrm{ml}$. The dimensions of the tank used for this visualization study were 90 $\times 40 \times 40 \mathrm{~cm}$. The top-bottom distance in each picture represents the scale, and is $28 \mathrm{~cm}$.

as well as a continuous change in their mutual distance; this results in an oscillation of the translation velocity and the horizontal size of the dipolar structure over a short time interval. These oscillations contain much more energy than in the linearly stratified case, because all the wave energy is now trapped within a relatively thin layer, and their effect on the vortex dipole is therefore more pronounced. Although waves of all modes and frequencies travel along the interface, because of conservation of the vertical component of the vorticity, the magnitude of the vorticity can only be changed when waves with mode 2 -or higher even modes-pass by. From video recordings the temporal evolution of the maximum width and displacement of the front of the dipole were measured. This method was adapted, rather than measuring the distance between the vortex centers of the dipole, because this distance is usually less well defined. In Fig. 13, the results for two different experiments with the same stratification are presented, which apparently show identical behavior. One can observe from this plot that the dipole's horizontal size and its displacement distance increase approximately linearly over the measured time interval, while showing shortperiod oscillations. Generally, the translation speed has decreased to zero when the dipole has reached its relative maximum size (see Fig. 13 at $t \simeq 105,125$, and $150 \mathrm{~s}$ ). Once the dipole reaches its minimum horizontal size the vortices have been stretched, and their centers have a smaller mutual distance. The combination of both leads to a higher translation velocity, resulting in a relatively rapid displacement of the front of the dipole (see Fig. 13 at $t \simeq 110,135$, and $155 \mathrm{~s}$ ). Subsequently, the dipole is squeezed, and its horizontal dimension increases until it reaches a maximum size again, and the same process repeats. Numerical simulations of the decomposed wavehorizontal vortex equations (Refs. 11 and 20) for the rem- 


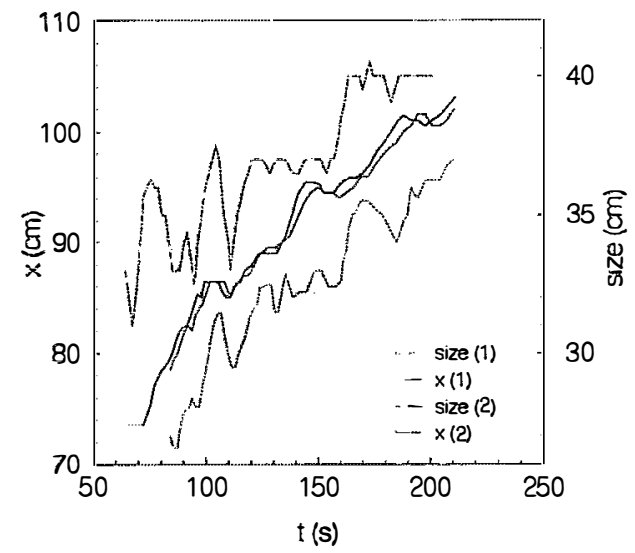

FIG. 13. Measured displacement of the dipolar front (solid lines) and size of the dipolar structure (dashed lines) for two different experiments. The experimental parameters for experiment 1 are $U_{0}=3.23 \mathrm{~m} / \mathrm{s}, V_{0}=4$ $\mathrm{ml}, \delta b=0.84 \mathrm{~m} / \mathrm{s}^{2}$, and for experiment 2 are $U_{0}=3.23 \mathrm{~m} / \mathrm{s}, V_{0}=5 \mathrm{ml}$, and $\delta b=0.84 \mathrm{~m} / \mathrm{s}^{2}$.

nant motions of a collapsed Kelvin-Hemlholtz billow in a two-layer shear flow have revealed similar oscillations in the vertical vorticity component when internal waves passed by. In these simulations the potential vorticity (defined as $\left.\omega \nabla \rho / \rho_{0}\right)$ did not oscillate, showing conservation of potential vertical vorticity and weak interaction between this vorticity and internal waves.

In order to compare the wave number and frequency of the internal waves with the oscillation frequency of the vortices, one can assume that the density profile of the two-layer fluid can be approximated by

$$
\rho=\bar{\rho} \exp \left(\frac{\delta b}{2 g} \tanh \frac{2\left(z-z_{i}\right)}{\delta z}\right),
$$

where $z_{i}$ and $\delta z$ are the level and thickness of the interface, respectively. When the wave amplitude is much smaller than the layer thickness, it is possible to obtain the dispersion relation as

$$
\begin{aligned}
k_{h}= & -\frac{(2 n+1) \omega^{2} / \delta z}{\omega^{2}-\delta b / \delta z}-\left[\left(\frac{(2 n+1) \omega^{2} / \delta z}{\omega^{2}-\delta b / \delta z}\right)^{2}\right. \\
& \left.-\frac{4\left(n^{2}+n\right) \omega^{2} / \delta z^{2}}{\omega^{2}-\delta b / \delta z}\right]^{1 / 2},
\end{aligned}
$$

where $n$ is the wave mode and $k_{h}$ is the wave number (see, e.g., Ref. 21). Waves interacting with the dipolar vortex should have at least a wavelength of twice the diameter of the dipole in order to stretch and squeeze the structure as a whole; from Fig. 13, it follows that the dipoles have a mean diameter of approximately $D=35 \mathrm{~cm}$, and thus waves with wave numbers $k_{h} \leqslant 2 \pi / 2 D \simeq 9 \mathrm{~m}^{-1}$ are the possible candidates. The frequency of these oscillations measured from Fig. 13 is approximately $\omega=0.25 \mathrm{rad} / \mathrm{s}$. Figure 14 shows that-according to relation (13)-the possible wave modes and wave numbers for this measured frequency are mode $n=1$ with $k_{h}=5 \mathrm{~m}^{-1}$ and mode $n=2$ with $k_{h}=9 \mathrm{~m}^{=-1}$. The wave number for mode 2 has the

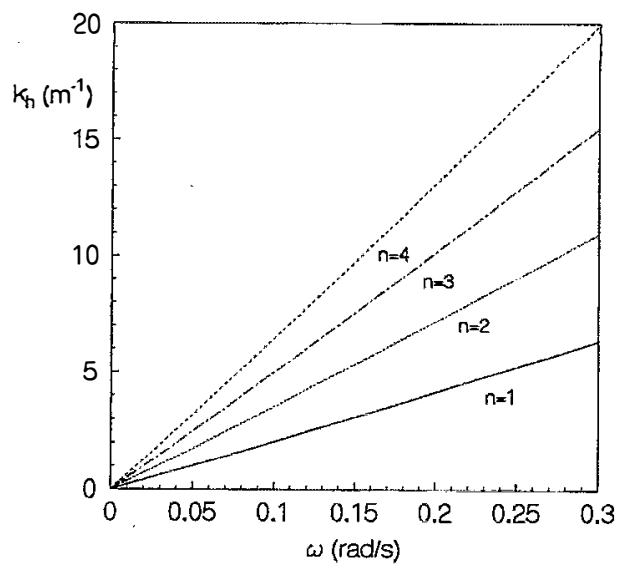

FIG. 14. The plot of Eq. (13) for the modes $n=1,2,3$, and 4 .

same frequency as the observed oscillation frequency $(0.25$ $\mathrm{rad} / \mathrm{s}$ ) of the dipolar vortex. Apparently, it is the wave mode 2 that causes the dipolar oscillation.

\section{CONCLUSIONS}

A laboratory study on the evolution of a turbulent region injected at the interface of a two-layer fluid was performed. An isolated blob of turbulent fluid in a twolayer fluid reaches its maximum height when the turbulent eddies in the blob come to a balance dictated by the vertical inertia forces of the eddies and the buoyancy forces associated with them. Before this occurs, the effect of stratification appears to be overshadowed by the inertia forces. After reaching this maximum height, the patch starts to collapse. This collapse leads to the formation of pancakelike eddies, which may be modulated by interfacial waves. The results can be summarized as follows.

(i) The vertical thickness of the region grows independent of the stratification for a time period $t_{c} \simeq 1.7$ $\left(V_{0} U_{0}^{2} / \delta b^{5}\right)^{1 / 8}$.

(ii) At $t_{c}$, the stratification becomes important and arrests the vertical growth; the vertical size of the patch under this critical condition is determined by a balance between vertical inertia and buoyancy forces of the eddies, and is given by $h_{c} \simeq 0.5\left(V_{0} U_{0}^{2} / \delta b\right)^{1 / 4}$.

(iii) Measurements indicate an initial increase of the Thorpe scale to a maximum value (coinciding with $h_{c}$ ), and a decrease thereafter. The decrease is slow, but is still faster than the decrease of the patch size, owing to the physical collapse. The ratio of the Thorpe scale to the maximum Thorpe displacement was found to remain constant over the time period investigated. The relationship between the Thorpe scale and the patch size was measured to be $L_{t} \approx 0.18 L_{p}$. The maximum Thorpe displacement was found to be given by $L_{t \max } / L_{p} \approx 0.45$. This is somewhat low compared to that observed in previous studies, and the development of stratification within the mixed layer during its evolution may be the reason for this observation.

(iv) The dipolar structure observed in the present twolayer experiments has the same appearance as in a linearly stratified fluid. However, in a two-layer fluid internal wave 
motions appear to be more energetic than in a linearly stratified fluid because of their trapping at the interface; thus the interaction between the waves and the vortices is strong. These internal waves are mainly of mode 2 , and subsequently stretch and squeeze the vortices, inducing oscillations of the translation speed and of the horizontal size of the dipole.

(v) The growth of a turbulent patch in two-layer and linearly stratified fluids (studied by FvHF) appear to be governed by similar dynamics, but some differences can be observed in detail. For example, in linearly stratified fluids the entrained fluid into the patch is subjected to mixing over the entire turbulent fluid column, whereas in the twolayer case a sharp interface may develop at the plane of symmetry, thus inhibiting the exchange of fluid between upper and lower halves of the patch. Thus the overturning distances can be smaller in the latter case, and the constants that appear in the different balances (scaling arguments) can be different for the two cases.

\section{ACKNOWLEDGMENTS}

We gratefully acknowledge the help of Dr. Prabath DeSilva for the use of software for density and Thorpescale calculations, Mr. Leonard Montenegro for technical assistance with the conductivity probes and Professor J. C. $R$. Hunt for his critical remarks on various aspects of this paper. One of us (JBF) gratefully acknowledges financial support by the Foundation for Fundamental Research on Matter (FOM) of the Netherlands Organization for Pure Research (NWO) and the U.S. Office of Naval Research (ONR), which made a seven-week visit to ASU possible.

'J. D. Woods, "Wave induced shear instability in the summer thermocline," J. Fluid Mech. 32, 791 (1968).

${ }^{2}$ M. C. Gregg, "Diapycnal mixing in the thermocline: A review," J. Geophys. Res. C 92, 5249 (1987).

${ }^{3} \mathrm{~K}$. A. Browning, "Structure of the atmosphere in the vicinity of large amplitude Kelvin Helmholtz billows," Q. J. R. Meteorol. Soc. 97, 283 (1971).

${ }^{4} \mathrm{G}$. Marmorino, "Observations of small-scale mixing processes in the seasonal thermocline. Part II: Wave breaking," J. Phys. Oceanogr. 17, 1348 (1987).

${ }^{5}$ H. J. S. Fernando, G. J. F. van Heijst, and S. V. Fonseka, "The evolution of an isolated turbulent region in a stratified fluid," submitted to $\mathrm{J}$. Fluid Mech.

${ }^{6}$ G. J. F. van Heijst and J. B. Flór, "Dipole formation and collisions in a stratified fluid," Nature 340, 212 (1989).

'S. I. Voropayev, Ya. D. Afanasyev, and I. A. Fillipov, "Horizontal jets and vortex dipoles in a stratified fluid," J. Fluid Mech. 227, 543 (1991).

${ }^{8}$ A. A. Townsend, The Structure of Turbulent Shear Flow (Cambridge U. P., Cambridge, 1976).

${ }^{9} \mathrm{H}$. Tennekes and J. L. Lumley, "A First Course in Turbulence," 6th ed. (MIT Press, Cambridge, 1980).

${ }^{10}$ T. M. Dillon, "Vertical overturns: A comparison of Thorpe and Ozmidov length scales," J. Geophys. Res. C 87, 9601 (1982).

"J. R. Riley, R. W. Metcalf, and M. A. Weissman, "Direct numerical simulations of homogeneous turbulence in density-stratified fluids," Proceedings of the AIP Conference on Nonlinear Properties of Internal Waves, edited by B. J. West (American Institute of Physics, New York, 1981 ), pp. 79-112.

${ }^{12} \mathrm{H}$. E. Gilreath and A. Brandt, "Experiments on the generation of internal waves in a stratified fluid," AIAA J. 23, 693 (1985).

${ }^{13}$ H. J. S. Fernando and R. R. Long, "On the nature of the entrainment interface of a two-layer fluid subjected to zero-mean-shear turbulence," J. Fluid Mech. 151, 21 (1985).

${ }^{14} \mathrm{H}$. J. S. Fernando, "The growth of a turbulent patch in a stratified fluid," J. Fluid Mech. 190, 55 (1988).

${ }^{15} \mathrm{~F}$. R. Mobbs, "Spreading and contraction at the boundaries of free turbulent flows," J. Fluid Mech. 33, 227 (1968).

${ }^{16}$ P. C. Manins, "Intrusion into a stratified fluid," J. Fluid Mech. 74, 547 (1976).

${ }^{17} \mathrm{E}$. C. Itsweire, "Measurements of vertical overtums in a stably stratified turbulent flow," Phys. Fluids 27, 764 (1984).

${ }^{18}$ L. P. D. DeSilva and H. J. S. Fernando, "Some aspects of turbulent mixing in a stratified turbulent patch," J. Fluid Mech. 140, 601 (1992).

${ }^{19} \mathrm{C}$. H. Gibson, "Laboratory, numerical and oceanic fossil turbulence in rotating and stratified flows," J. Geophys. Res. C 96, 12549 (1987).

${ }^{20} \mathrm{C}$. Staquet and J. J. Riley, "On the velocity field associated with potential vorticity," Dyn. Atmos. Oceans 14, 93 (1989).

${ }^{21}$ W. Krauss, Internal waves, The Methods of Theoretical Oceanography (Gebrüder Borntraeger, Berlin, 1966), Vol. 2. 